\title{
Small-scale screening of anticancer drugs acting specifically on neural stem/progenitor cells derived from human induced pluripotent stem cells using a time-course cytotoxicity test
}

\author{
Hayato Fukusumi $^{1}$, Yukako Handa $^{2}$, Tomoko Shofuda ${ }^{1}$, Yonehiro Kanemura ${ }^{\text {Corresp. }{ }^{2,3,4}}$ \\ 1 Division of Stem Cell Research, Institute for Clinical Research, Osaka National Hospital, National Hospital Organization, Osaka, Japan \\ 2 Division of Regenerative Medicine, Institute for Clinical Research, Osaka National Hospital, National Hospital Organization, Osaka, Japan \\ 3 Department of Neurosurgery, Osaka National Hospital, National Hospital Organization, Osaka, Japan \\ 4 Department of Physiology, Keio University School of Medicine, Tokyo, Japan \\ Corresponding Author: Yonehiro Kanemura \\ Email address: kanemura@onh.go.jp
}

Since the development of human induced pluripotent stem cells (hiPSCs), various types of hiPSC-derived cells have been established for regenerative medicine and drug development. Neural stem/progenitor cells (NSPCs) derived from hiPSCs (hiPSC-NSPCs) have shown benefits for regenerative therapy of the central nervous system. However, owing to their intrinsic proliferative potential, therapies using transplanted hiPSC-NSPCS carry an inherent risk of undesired growth in vivo. Therefore, it is important to find cytotoxic drugs that can specifically target overproliferative transplanted hiPSC-NSPCS without damaging the intrinsic in vivo stem-cell system. Here, we examined the chemosensitivity of hiPSC-NSPCs and human neural tissue-derived NSPCs (hN-NSPCs) to the general anticancer drugs cisplatin, etoposide, mercaptopurine, and methotrexate. A time-course analysis of neurospheres in a microsphere array identified cisplatin and etoposide as fast-acting drugs, and mercaptopurine and methotrexate as slow-acting drugs. Notably, the slow-acting drugs were eventually cytotoxic to hiPSC-NSPCs but not to hN-NSPCs, a phenomenon not evident in the conventional endpoint assay on day 2 of treatment. Our results indicate that slow-acting drugs can distinguish hiPSC-NSPCs from hN-NSPCs and may provide an effective backup safety measure in stem-cell transplant therapies. 


\section{Small-scale screening of anticancer drugs acting specifically}

2 on neural stem/progenitor cells derived from human induced

\section{3 pluripotent stem cells using a time-course cytotoxicity test}

4 Running head: Screening of drugs for hiPSC-NSPCs

5 Hayato Fukusumi ${ }^{1}$, Yukako Handa ${ }^{2}$, Tomoko Shofuda ${ }^{1}$, Yonehiro Kanemura ${ }^{2,3,4}$

$7{ }^{1}$ Division of Stem Cell Research, Institute for Clinical Research, Osaka National Hospital,

8 National Hospital Organization, Osaka 540-0006, Japan

$9 \quad 2$ Division of Regenerative Medicine, Institute for Clinical Research, Osaka National Hospital,

10 National Hospital Organization, Osaka 540-0006, Japan

$11{ }^{3}$ Department of Neurosurgery, Osaka National Hospital, National Hospital Organization, Osaka

12 540-0006, Japan

$13{ }^{4}$ Department of Physiology, Keio University School of Medicine, Tokyo 160-8582, Japan

15 Corresponding Author:

16 Yonehiro Kanemura, $\mathrm{MD}, \mathrm{PhD}^{2,3,4}$

17 Email address: kanemura@onh.go.jp 


\section{Abstract}

20 Since the development of human induced pluripotent stem cells (hiPSCs), various types of

21 hiPSC-derived cells have been established for regenerative medicine and drug development.

22 Neural stem/progenitor cells (NSPCs) derived from hiPSCs (hiPSC-NSPCs) have shown benefits

23 for regenerative therapy of the central nervous system. However, owing to their intrinsic

24 proliferative potential, therapies using transplanted hiPSC-NSPCs carry an inherent risk of

25 undesired growth in vivo. Therefore, it is important to find cytotoxic drugs that can specifically

26 target overproliferative transplanted hiPSC-NSPCs without damaging the intrinsic in vivo stem-

27 cell system. Here, we examined the chemosensitivity of hiPSC-NSPCs and human neural tissue-

28 derived NSPCs (hN-NSPCs) to the general anticancer drugs cisplatin, etoposide, mercaptopurine,

29 and methotrexate. A time-course analysis of neurospheres in a microsphere array identified

30 cisplatin and etoposide as fast-acting drugs, and mercaptopurine and methotrexate as slow-acting

31 drugs. Notably, the slow-acting drugs were eventually cytotoxic to hiPSC-NSPCs but not to hN-

32 NSPCs, a phenomenon not evident in the conventional endpoint assay on day 2 of treatment. Our

33 results indicate that slow-acting drugs can distinguish hiPSC-NSPCs from hN-NSPCs and may

34 provide an effective backup safety measure in stem-cell transplant therapies. 


\section{Introduction}

36 Since the development of human induced pluripotent stem cells (hiPSCs) (Takahashi et al. 2007;

37 Yu et al. 2007), various types of hiPSC-derived cells have been established that can be used in

38 regenerative medicine and drug development, while avoiding many of the ethical issues and

39 technical difficulties involved with human tissue-derived cells. Human iPSC-derived neural

40 stem/progenitor cells (hiPSC-NSPCs) (Fujimoto et al. 2012; Kobayashi et al. 2012; Oki et al.

41 2012; Tornero et al. 2013) and human fetal neural tissue-derived NSPCs (hN-NSPCs) (Ishibashi

42 et al. 2004; Iwanami et al. 2005; Ogawa et al. 2002) have proven beneficial in treating various

43 central nervous system diseases and injuries. However, the intrinsic proliferative potential of

44 hiPSC-NSPCs, which makes them promising sources for large numbers of cells in vitro, can be a

45 double-edged sword in vivo: transplanted cells can proliferate excessively before terminal

46 differentiation in specific microenvironments. Although such undesired proliferation has not

47 generally produced teratomas, malignant carcinogenesis, or other serious adverse events (Nori et

48 al. 2015; Sugai et al. 2016), this inherent potential suggests the need for backup safety measures

49 for stem cell-based therapies.

One strategy for reducing the risk of overgrowth is to transduce a gene that can induce

51 apoptosis, such as herpes simplex virus truncated thymidine kinase (HSV-tk) activated by

52 ganciclovir (Cao et al. 2007) or a caspase-based artificial cell-death switch (iCaspase-9) 
53 activated by AP20187 (Krishnamurthy et al. 2010), into the stem-cell genome. However,

54 inserting exogenous genes into the donor-cell genome contradicts the purpose of integration-free

55 hiPSCs, which are generated to minimize the risk of genetic modification or transgene re-

56 activation, and transgenic strategies may create new risks despite the use of 'genomic safe

57 harbors' for insertions in the human genome. Another strategy is to use drugs to suppress the in

58 vivo overgrowth of transplanted cells; for instance, pretreating hiPSC-NSPCs with a $\gamma$-secretase

59 inhibitor inhibits Notch signaling, which is required for maintaining NSPC stemness (Okubo et

60 al. 2016). However, a single treatment prior to transplantation may not be sufficient to overcome

61 the cells' growth potential, and cannot regulate cell growth after transplantation. Therefore, a

62 useful backup safety measure would be a method to chemically ablate transplanted cells,

63 preferably with a cytotoxic drug that specifically acts on transplanted hiPSC-NSPCs but not

64 tissue-resident NSPCs.

65 In this study, we assessed four approved anticancer drugs, two cytotoxic (cisplatin and

66 etoposide) and two cytostatic (mercaptopurine and methotrexate), as candidates for suppressing

67 the overgrowth of non-transgenic stem cells in vivo.

68 Although the efficacy of candidate drugs has conventionally been evaluated by cell-destructive

69 methods, such as MTT or ATP assays, these methods cannot assess the effects of a drug on the

70 same cell population over time. To address this, previous studies have assessed the time-course 
71 of pharmacological effects using cell-nondestructive methods, such as measurement of changes

72 in impedance in two-dimensional (2D) adherent cell cultures (Caviglia et al. 2015) and image-

73 based measurement of the spheroid size in three-dimensional (3D) cultures of various cell types,

74 including glioma cells (Vinci et al. 2012), hepatocytes (Bell et al. 2016), and cardiomyocytes

75 (Beauchamp et al. 2015). It is known that 3D culture systems mimic the in vivo environment

76 more effectively than 2D culture systems (Achilli et al. 2012; Pampaloni et al. 2007). Therefore,

77 the present study used a conventional endpoint assay on day 2 of the treatment and a 7-day time-

78 course cytotoxicity test to determine the effects of cisplatin, etoposide, mercaptopurine, and

79 methotrexate on 3D neurospheres derived from hiPSC-NSPCs and hN-NSPCs, which are

80 considered to mimic the in vivo stem cell system. 


\section{Materials and Methods}

\section{Ethics statement}

84 This study was conducted in accordance with the principles of the Declaration of Helsinki. The

85 use of hN-NSPCs and hiPSCs was approved by the Osaka National Hospital hN-NSPCs and

86 hiPSCs ethics committee (Nos. 110, 120, and 146).

\section{Cell lines}

88 We used two hN-NSPC lines (oh-NSC-3-fb and oh-NSC-7-fb) (Kanemura et al. 2002) and two

89 hiPSC (201B7)-derived NSPC lines: the DSM line, which was established using the single

90 SMAD-inhibition method with the Noggin alternative dorsomorphin (DSM) (Shofuda et al.

91 2013), and the dSMAD line, which was established by the dual SMAD-inhibition method with

92 DSM and SB431542 (Fukusumi et al. 2016).

93 Cell culture

94 The hN-NSPCs and hiPSC-NSPCs were propagated as neurospheres in Dulbecco's Modified

95 Eagle's Medium (DMEM)/F12 (D8062; Sigma-Aldrich, St. Louis, MO, USA) with 15 mM

96 HEPES (Sigma-Aldrich), epidermal growth factor (EGF, 20 ng/mL; PeproTech, Rocky Hill, NJ,

97 USA), fibroblast growth factor 2 (FGF2, $20 \mathrm{ng} / \mathrm{mL}$; PeproTech), leukemia inhibitory factor (LIF,

9810 ng/mL; Millipore, Billerica, MA, USA), B27 supplement (B27, 2\%; Thermo Fisher Scientific,

99 Waltham, MA, USA), and heparin (5 $\mu \mathrm{g} / \mathrm{mL}$; Sigma-Aldrich) (Fukusumi et al. 2016; Kanemura 
100 et al. 2002; Shofuda et al. 2013). For hN-NSPCs, half of the medium was changed once a week.

101 The neurospheres were dissociated into single cells every 14 days by incubating them with

$1020.05 \%$ trypsin/EDTA (Thermo Fisher Scientific) at $37^{\circ} \mathrm{C}$ for $20 \mathrm{~min}$, after which soybean trypsin

103 inhibitor (Roche, Basel, Switzerland) was added to stop the enzyme activity. The cells were then

104 resuspended in 50\% fresh medium plus 50\% conditioned medium at a density of $1 \times 10^{5}$

105 cells/mL (Kanemura et al. 2002). For hiPSC-NSPCs, the medium was changed every 3-5 days.

106 The cells were passaged every 10-12 days using Accutase (Innovative Cell Technologies, San

107 Diego, CA, USA) at $37^{\circ} \mathrm{C}$ for $10 \mathrm{~min}$ for single-cell dissociation, after which the cells were

108 resuspended in $100 \%$ fresh medium at a density of $1 \times 10^{5}$ cells/mL (Fukusumi et al. 2016;

109 Shofuda et al. 2013).

110 Drug preparation

111 Cisplatin (Sigma-Aldrich), etoposide (Sigma-Aldrich), mercaptopurine (Sigma-Aldrich), and

112 methotrexate (LKT Laboratories, St. Paul, MN, USA) were dissolved in dimethyl sulfoxide

113 (DMSO) to generate $100 \mathrm{mM}$ stock solutions.

114 Endpoint (ATP) assay

115 The neurospheres were dissociated into single cells and seeded into 96-well plates at a density of

$1163 \times 10^{4}$ cells/well (day -1$)$. On day 0 , cisplatin, etoposide, and methotrexate were applied at 0 ,

$1170.1,0.3,1,3,10,30$, and $100 \mu \mathrm{M}$, and mercaptopurine was applied at $0,1,3,10,30,100,300$, 
118 and $1000 \mu \mathrm{M}(0 \mu \mathrm{M}$ indicates DMSO only). ATP content was assayed after $48 \mathrm{~h}$ with CellTiter-

119 Glo reagent (Promega, Madison, WI, USA) according to the manufacturer's instructions. Briefly,

$12050 \mu \mathrm{L}$ of CellTiter-Glo was added to wells containing $50 \mu \mathrm{L}$ of medium. The plates were shaken

121 for $2 \mathrm{~min}$ and incubated for $20 \mathrm{~min}$ at room temperature, and luminescence was determined on a

122 Wallac 1420 ARVOsx (PerkinElmer, Norwalk, CT, USA).

123 Time-course cytotoxicity test using a microsphere array (MSA)

124 The MSA (MSE24-CA300, 652 microwells/array; STEM Biomethod, Fukuoka, Japan) was set

125 into one well of a 24-well plate. The neurospheres were dissociated into single cells and seeded

126 into the MSA at a density of 200 cells/microwell (day -1). On day 0 , the pretreatment state was

127 recorded by phase-contrast images of neurospheres in the MSA microwells (Fig. 1a and Fig. 1b),

128 and etoposide $(0,0.1,1$, and $10 \mu \mathrm{M})$ or cisplatin, mercaptopurine, or methotrexate $(0,1,10$, and

$129100 \mu \mathrm{M}$ ) was applied (control, low, middle, and high concentrations, respectively). This medium

130 was not replaced during the 7-day experiment. Phase-contrast images were captured on days 1, 2,

1313 , and 7 to monitor neurospheres in the microwells (Fig. 1a and Fig. 1b), and the projected areas

132 of neurospheres (Mori et al. 2006) were measured using a pen tablet (Intuos, CTH-480; Wacom,

133 Saitama, Japan) with the TrakEM2 plugin (Cardona et al. 2012) in ImageJ (Fiji package)

134 (Schindelin et al. 2012; Schindelin et al. 2015). Microwells containing multiple neurospheres on

135 day 0 were excluded from analysis. Outliers in a boxplot of neurosphere sizes on day 0 were also 
136 excluded. At each time-point, the neurosphere size in the presence of each treatment was

137 expressed as a percentage of the day 0 value. These data were then expressed as a percentage of

138 the respective DMSO control.

139 Statistical analysis

140 For the endpoint assay (Fig. 2), predicted dose-response curves and 50\% inhibitory concentration

$141\left(\mathrm{IC}_{50}\right)$ values were obtained using the four-parameter log-logistic (LL2.4) and ED functions,

142 respectively, of the drc package (Ritz et al. 2015) in R (https://www.R-project.org/). For the

143 time-course cytotoxicity test, changes in neurosphere size relative to those in the DMSO controls

144 (Fig. 3) and day 0 neurospheres (Fig. S1) were analyzed with the four-parameter logistic model

145 (L.4) and the Brain-Cousens five-parameter model (BC.5) of the drc package in R, respectively.

146 Data from the endpoint assay (Fig. 2) and time-course cytotoxicity test (Fig. 3 and Fig. S1) were

147 plotted with $95 \%$ confidence intervals $(95 \% \mathrm{CI})$. 


\section{Results}

150 The $\mathrm{IC}_{50}$ of cisplatin, etoposide, mercaptopurine, and methotrexate in the two hiPSC-NSPC cell

151 lines (DSM and dSMAD) and the two hN-NSPC cell lines (oh-NSC-3-fb and oh-NSC-7-fb; Fig.

1522 and Table 1) was determined by endpoint (ATP) assay. Cisplatin and etoposide were

153 preferentially toxic to hN-NSPCs and hiPSC-NSPCs, respectively. Interestingly, the two hiPSC-

154 NSPC lines differed in sensitivity to these drugs (Table 1). Mercaptopurine was highly toxic to

155 both types of NSPCs, while methotrexate had almost no effect on either type even at high

156 concentrations. Of the two hiPSC-NSPC lines, DSM was significantly more resistant to the drugs. To follow the real-time effects of these drugs after treatment, we conducted a simple time-course cytotoxicity test using an MSA (Fig. 1a and 1b). Similar to the ATP assay, we assessed the effect of cisplatin, etoposide, mercaptopurine, and methotrexate at four dosage levels in the two hiPSC-NSPC and two hN-NSPCs lines, in this case by measuring neurosphere

161 size on each day for 7 days (Fig. 3 and Table S1). Because neurosphere size of the DMSO

162 control increased during the 7-day assay (Fig. S1), smaller neurosphere sizes of the drug

163 treatment group than those of the DMSO control indicate cytotoxicity of drugs.

164 Compared to the DMSO control, cisplatin showed toxic effects in hiPSC-NSPCs at low

165 to high concentrations and in hN-NSPCs at middle and high concentrations. Notably, high 166 concentrations of cisplatin killed hiPSC-NSPC neurospheres, as indicated by their disappearance 
167 (Fig. S1 shows changes in neurosphere size relative to day 0), whereas hN-NSPC neurospheres

168 were present throughout the assay period (Fig. S1). Etoposide also affected the neurosphere size

169 in both hiPSC-NSPCs and hN-NSPCs, but it showed earlier and stronger toxicity at lower

170 concentrations compared to cisplatin. However, the hN-NSPC neurosphere size remained

171 constant even at the highest concentration of etoposide or cisplatin (Fig. S1). Thus, there was a

172 clear difference between hiPSC-NSPCs and hN-NSPCs at the highest concentrations of both

173 cisplatin and etoposide. Moreover, low concentrations of etoposide affected the neurosphere size

174 in dSMAD and oh-NSC-7-fb cells more strongly than that in DSM or oh-NSC-3-fb cells,

175 respectively. Thus, the preferential toxicity of the drug differed not only between the two types

176 of NSPCs, but also between two cell lines of the same type.

177 Mercaptopurine and methotrexate, even at the highest concentrations, were only mildly

178 toxic to hN-NSPCs and did not stop their growth (Fig. S1). However, hiPSC-NSPCs were

179 affected by both mercaptopurine and methotrexate. At low concentrations, methotrexate was

180 noticeably more toxic to hiPSC-NSPCs than was mercaptopurine. Unlike cisplatin and etoposide,

181 mercaptopurine and methotrexate had only a limited effect at even the highest concentrations

182 until day 3, which allowed the growth of larger neurospheres (Fig. S1). As with cisplatin and

183 etoposide, mercaptopurine and methotrexate proved to be highly cytotoxic by the end of the

184 assay. From these results, cisplatin and etoposide can be classified as fast-acting drugs with early 
185 cellular toxicity, while mercaptopurine and methotrexate can be classified as slow-acting drugs

186 with later toxicity. 
187 Discussion

188

Although hiPSC-NSPC transplantation is effective for treating spinal cord injury and

189

stroke, the use of stem cells poses certain risks due to their intrinsic proliferative potential. In

190

particular, the artificial generation of hiPSCs may cause genetic and epigenetic abnormalities,

which could potentially increase the risk of tumorigenesis (Nagoshi \& Okano 2017). These risks

192

can be reduced prior to transplantation by inserting a 'suicide gene' into the donor cells, or by

193

pretreating the donor cells with inhibitors that reduce their stemness and direct differentiation.

194

However, neither of these strategies is ideal. Transgenes can potentially introduce new risks via

genome modification. Although pretreatment can reduce donor-cell stemness, we have not yet

found a way to deal with the overgrowth of grafted cells after transplantation. Thus, as a backup

safety measure for stem-cell therapies, it is essential to identify drugs that act specifically on the

grafted cells, but not on resident stem cells. To this end, we conducted a small-scale screening of

four anticancer drugs and examined their effect on hiPSCs-NSPCs and on hN-NSPCs, which are

considered to mimic the resident stem cells in the host body.

Based on ATP assay results, we classified the four drugs as follows: (1) more toxic to

hN-NSPCs than hiPSC-NSPCs (cisplatin), (2) more toxic to hiPSC-NSPCs than hN-NSPCs

203 (etoposide), (3) similar toxic effects on hiPSC-NSPCs and hN-NSPCs (mercaptopurine), and (4)

204 almost no effect on hiPSC-NSPCs or hN-NSPCs (methotrexate). These results identified 
205 etoposide as a candidate backup safety measure for stem cell-based therapies, since it was

206 selectively toxic to hiPSC-NSPCs. However, the dose may need to be adjusted for individual cell

207 lines, since different lines of the same type of NSPC differed in sensitivity to etoposide.

208 Although an ATP assay is useful for characterizing drugs based on dose-response curves and

$209 \mathrm{IC}_{50}$ values, the long-term monitoring of transplanted cells is necessary after treatment in vivo.

210 In this study, we monitored the effects of anticancer drugs on neurosphere size in vitro

211 for a period of 7 days after treatment. Based on these results, we classified cisplatin and

212 etoposide as fast-acting drugs with early cytotoxicity, and mercaptopurine and methotrexate as

213 slow-acting drugs with late cytotoxicity; this classification is consistent with the drug categories.

214 Cisplatin and etoposide are cytotoxic drugs that act directly by alkylating DNA and inhibiting

215 topoisomerase, respectively, whereas mercaptopurine and methotrexate are cytostatic drugs that

216 inhibit IMP dehydrogenase and dihydrofolate reductase, respectively. Compared to hiPSC-

217 NSPCs, the hN-NSPCs were more resistant to high concentrations of cisplatin or etoposide; this

218 difference might be due to the different developmental stages of the cells. In fact, hiPSC-NSPCs

219 recapitulate regular neural development along with cell proliferation after transplantation (Sugai

220 et al. 2016), and this characteristic will likely be useful for developing drugs that specifically

221 target transplanted cells. The presence of mercaptopurine and methotrexate, which are cytostatic,

222 eventually induced death in hiPSC-NSPCs, but only mildly limited hN-NSPC growth during the 
223 7-day assay. Mercaptopurine and the cytotoxic drugs cisplatin and etoposide decreased the ATP

224 level in both hN-NSPCs and hiPSC-NSPCs on day 2 of treatment (Fig. 2). Although ATP level is

225 a useful index of cell viability, mercaptopurine-mediated inhibition of de novo purine synthesis

226 might also reduce the ATP level in the absence of cell death, in contrast to other cytotoxic drugs.

227 However, the effect of cytostatic drugs distinguished hN-NSPCs and hiPSC-NSPCs in the time-

228 course assay (Fig. 3). Therefore, we need to reassess cytostatic drugs from the viewpoint of their

229 time-dependent action. Our findings indicate that methotrexate is preferable to mercaptopurine as

230 a candidate safety measure for hiPSC-NSPC transplantation because it was cytotoxic even at low

231 concentrations.

232 This study has certain limitations. First, the mechanisms underlying the late toxicity of

233 cytostatic drugs on hiPSC-NSPCs are unknown, although hN-NSPCs are reported to express

234 high levels of ABCB1 transporter (Islam et al. 2005; Yamamoto et al. 2009), which may

235 contribute to a development of tolerance to slow-acting drugs. Further studies are needed to

236 elucidate the mechanisms underlying the selective cytotoxic effects of cytostatic drugs on hiPSC-

237 NSPCs. Second, these effects were obtained in vitro and therefore await confirmation in vivo. 


\section{Conclusion}

239 Based on a 7-day time-course cytotoxicity test, we classified four anticancer drugs as fast-acting

240 or slow-acting. We found that the slow-acting drugs affected hiPSCs-NSPCs and hN-NSPCs

241 differently, which was not evident in a conventional ATP assay performed on day 2. As hN-

242 NSPCs were more tolerant of slow-acting drugs than hiPSC-NSPCs, we propose that slow-acting

243 drugs such as methotrexate may provide drug candidates for backup safety measures to prevent

244 the undesirable proliferation of hiPSC-NSPCs after transplantation therapies. 


\section{Acknowledgments}

248 The authors thank Ms. Ai Takada, Ms. Miho Sumida, Ms. Ema Yoshioka, Ms. Yui Inazawa, and

249 Mr. Daisuke Kanematsu for technical support. 


\section{References}

252 Achilli TM, Meyer J, and Morgan JR. 2012. Advances in the formation, use and understanding

253 of multi-cellular spheroids. Expert Opinion on Biological Therapy 12:1347-1360.

$254 \quad 10.1517 / 14712598.2012 .707181$

255 Beauchamp P, Moritz W, Kelm JM, Ullrich ND, Agarkova I, Anson BD, Suter TM, and

256 Zuppinger C. 2015. Development and Characterization of a Scaffold-Free 3D Spheroid Model of

257 Induced Pluripotent Stem Cell-Derived Human Cardiomyocytes. Tissue Eng Part C Methods

$258 \quad 21: 852-861.10 .1089 /$ ten.TEC.2014.0376

259 Bell CC, Hendriks DF, Moro SM, Ellis E, Walsh J, Renblom A, Fredriksson Puigvert L, Dankers

260 AC, Jacobs F, Snoeys J, Sison-Young RL, Jenkins RE, Nordling A, Mkrtchian S, Park BK,

261 Kitteringham NR, Goldring CE, Lauschke VM, and Ingelman-Sundberg M. 2016.

262 Characterization of primary human hepatocyte spheroids as a model system for drug-induced

263 liver injury, liver function and disease. Scientific Reports 6:25187. 10.1038/srep25187

264 Cao F, Drukker M, Lin S, Sheikh AY, Xie X, Li Z, Connolly AJ, Weissman IL, and Wu JC.

265 2007. Molecular imaging of embryonic stem cell misbehavior and suicide gene ablation. Cloning

266 and Stem Cells 9:107-117. 10.1089/clo.2006.0E16 
267 Cardona A, Saalfeld S, Schindelin J, Arganda-Carreras I, Preibisch S, Longair M, Tomancak P,

268 Hartenstein V, and Douglas RJ. 2012. TrakEM2 software for neural circuit reconstruction. PloS

269 One 7:e38011. 10.1371/journal.pone.0038011

270 Caviglia C, Zor K, Canepa S, Carminati M, Larsen LB, Raiteri R, Andresen TL, Heiskanen A,

271 and Emneus J. 2015. Interdependence of initial cell density, drug concentration and exposure

272 time revealed by real-time impedance spectroscopic cytotoxicity assay. Analyst 140:3623-3629.

$273 \quad 10.1039 / \mathrm{c} 5 \mathrm{an} 00097 \mathrm{a}$

274 Fujimoto Y, Abematsu M, Falk A, Tsujimura K, Sanosaka T, Juliandi B, Semi K, Namihira M,

275 Komiya S, Smith A, and Nakashima K. 2012. Treatment of a mouse model of spinal cord injury

276 by transplantation of human induced pluripotent stem cell-derived long-term self-renewing

277 neuroepithelial-like stem cells. Stem Cells 30:1163-1173. 10.1002/stem.1083

278 Fukusumi H, Shofuda T, Bamba Y, Yamamoto A, Kanematsu D, Handa Y, Okita K, Nakamura

279 M, Yamanaka S, Okano H, and Kanemura Y. 2016. Establishment of human neural progenitor

280 cells from human induced pluripotent stem cells with diverse tissue origins. Stem Cells Int

$281 \quad 2016: 7235757.10 .1155 / 2016 / 7235757$

282 Ishibashi S, Sakaguchi M, Kuroiwa T, Yamasaki M, Kanemura Y, Shizuko I, Shimazaki T,

283 Onodera M, Okano H, and Mizusawa H. 2004. Human neural stem/progenitor cells, expanded in 
284 long-term neurosphere culture, promote functional recovery after focal ischemia in Mongolian

285 gerbils. Journal of Neuroscience Research 78:215-223. 10.1002/jnr.20246

286 Islam MO, Kanemura Y, Tajria J, Mori H, Kobayashi S, Shofuda T, Miyake J, Hara M,

287 Yamasaki M, and Okano H. 2005. Characterization of ABC transporter ABCB1 expressed in

288 human neural stem/progenitor cells. FEBS Letters 579:3473-3480. 10.1016/j.febslet.2005.05.019

289 Iwanami A, Kaneko S, Nakamura M, Kanemura Y, Mori H, Kobayashi S, Yamasaki M,

290 Momoshima S, Ishii H, Ando K, Tanioka Y, Tamaoki N, Nomura T, Toyama Y, and Okano H.

291 2005. Transplantation of human neural stem cells for spinal cord injury in primates. Journal of

292 Neuroscience Research 80:182-190. 10.1002/jnr.20436

293 Kanemura Y, Mori H, Kobayashi S, Islam O, Kodama E, Yamamoto A, Nakanishi Y, Arita N,

294 Yamasaki M, Okano H, Hara M, and Miyake J. 2002. Evaluation of in vitro proliferative activity

295 of human fetal neural stem/progenitor cells using indirect measurements of viable cells based on

296 cellular metabolic activity. J Neurosci Res 69:869-879. 10.1002/jnr.10377

297 Kobayashi Y, Okada Y, Itakura G, Iwai H, Nishimura S, Yasuda A, Nori S, Hikishima K,

298 Konomi T, Fujiyoshi K, Tsuji O, Toyama Y, Yamanaka S, Nakamura M, and Okano H. 2012.

299 Pre-evaluated safe human iPSC-derived neural stem cells promote functional recovery after

300 spinal cord injury in common marmoset without tumorigenicity. PloS One 7:e52787.

301 10.1371/journal.pone.0052787 
302 Krishnamurthy S, Dong Z, Vodopyanov D, Imai A, Helman JI, Prince ME, Wicha MS, and Nor

303 JE. 2010. Endothelial cell-initiated signaling promotes the survival and self-renewal of cancer

304 stem cells. Cancer Research 70:9969-9978. 10.1158/0008-5472.CAN-10-1712

305 Mori H, Ninomiya K, Kino-oka M, Shofuda T, Islam MO, Yamasaki M, Okano H, Taya M, and

306 Kanemura Y. 2006. Effect of neurosphere size on the growth rate of human neural

307 stem/progenitor cells. J Neurosci Res 84:1682-1691. 10.1002/jnr.21082

308 Nagoshi N, and Okano H. 2017. iPSC-derived neural precursor cells: potential for cell

309 transplantation therapy in spinal cord injury. Cell Mol Life Sci. 10.1007/s00018-017-2676-9

310 Nori S, Okada Y, Nishimura S, Sasaki T, Itakura G, Kobayashi Y, Renault-Mihara F, Shimizu A,

311 Koya I, Yoshida R, Kudoh J, Koike M, Uchiyama Y, Ikeda E, Toyama Y, Nakamura M, and

312 Okano H. 2015. Long-term safety issues of iPSC-based cell therapy in a spinal cord injury

313 model: oncogenic transformation with epithelial-mesenchymal transition. Stem Cell Reports

314 4:360-373. 10.1016/j.stemcr.2015.01.006

315 Ogawa Y, Sawamoto K, Miyata T, Miyao S, Watanabe M, Nakamura M, Bregman BS, Koike M,

316 Uchiyama Y, Toyama Y, and Okano H. 2002. Transplantation of in vitro-expanded fetal neural

317 progenitor cells results in neurogenesis and functional recovery after spinal cord contusion injury

318 in adult rats. Journal of Neuroscience Research 69:925-933. 10.1002/jnr.10341 
319 Oki K, Tatarishvili J, Wood J, Koch P, Wattananit S, Mine Y, Monni E, Tornero D, Ahlenius H,

320 Ladewig J, Brustle O, Lindvall O, and Kokaia Z. 2012. Human-induced pluripotent stem cells

321 form functional neurons and improve recovery after grafting in stroke-damaged brain. Stem Cells

$322 \quad 30: 1120-1133.10 .1002 /$ stem.1104

323 Okubo T, Iwanami A, Kohyama J, Itakura G, Kawabata S, Nishiyama Y, Sugai K, Ozaki M, Iida

324 T, Matsubayashi K, Matsumoto M, Nakamura M, and Okano H. 2016. Pretreatment with a

325 gamma-Secretase Inhibitor Prevents Tumor-like Overgrowth in Human iPSC-Derived

326 Transplants for Spinal Cord Injury. Stem Cell Reports 7:649-663. 10.1016/j.stemcr.2016.08.015

327 Pampaloni F, Reynaud EG, and Stelzer EH. 2007. The third dimension bridges the gap between

328 cell culture and live tissue. Nature Reviews: Molecular Cell Biology 8:839-845.

$329 \quad 10.1038 / \mathrm{nrm} 2236$

330 Ritz C, Baty F, Streibig JC, and Gerhard D. 2015. Dose-response analysis using R. PloS One

331 10:e0146021. 10.1371/journal.pone.0146021

332 Schindelin J, Arganda-Carreras I, Frise E, Kaynig V, Longair M, Pietzsch T, Preibisch S,

333 Rueden C, Saalfeld S, Schmid B, Tinevez JY, White DJ, Hartenstein V, Eliceiri K, Tomancak P,

334 and Cardona A. 2012. Fiji: an open-source platform for biological-image analysis. Nature

335 Methods 9:676-682. 10.1038/nmeth.2019 
336 Schindelin J, Rueden CT, Hiner MC, and Eliceiri KW. 2015. The ImageJ ecosystem: an open

337 platform for biomedical image analysis. Molecular Reproduction and Development 82:518-529.

$338 \quad 10.1002 / \mathrm{mrd} .22489$

339 Shofuda T, Fukusumi H, Kanematsu D, Yamamoto A, Yamasaki M, Arita N, and Kanemura Y.

340 2013. A method for efficiently generating neurospheres from human-induced pluripotent stem

341 cells using microsphere arrays. Neuroreport 24:84-90. 10.1097/WNR.0b013e32835cb677

342 Sugai K, Fukuzawa R, Shofuda T, Fukusumi H, Kawabata S, Nishiyama Y, Higuchi Y, Kawai K,

343 Isoda M, Kanematsu D, Hashimoto-Tamaoki T, Kohyama J, Iwanami A, Suemizu H, Ikeda E,

344 Matsumoto M, Kanemura Y, Nakamura M, and Okano H. 2016. Pathological classification of

345 human iPSC-derived neural stem/progenitor cells towards safety assessment of transplantation

346 therapy for CNS diseases. Molecular Brain 9:85. 10.1186/s13041-016-0265-8

347 Takahashi K, Tanabe K, Ohnuki M, Narita M, Ichisaka T, Tomoda K, and Yamanaka S. 2007.

348 Induction of pluripotent stem cells from adult human fibroblasts by defined factors. Cell

349 131:861-872. 10.1016/j.cell.2007.11.019

350 Tornero D, Wattananit S, Gronning Madsen M, Koch P, Wood J, Tatarishvili J, Mine Y, Ge R,

351 Monni E, Devaraju K, Hevner RF, Brustle O, Lindvall O, and Kokaia Z. 2013. Human induced

352 pluripotent stem cell-derived cortical neurons integrate in stroke-injured cortex and improve

353 functional recovery. Brain 136:3561-3577. 10.1093/brain/awt278 
354 Vinci M, Gowan S, Boxall F, Patterson L, Zimmermann M, Court W, Lomas C, Mendiola M,

355 Hardisson D, and Eccles SA. 2012. Advances in establishment and analysis of three-dimensional

356 tumor spheroid-based functional assays for target validation and drug evaluation. BMC Biology

357 10:29. 10.1186/1741-7007-10-29

358 Yamamoto A, Shofuda T, Islam MO, Nakamura Y, Yamasaki M, Okano H, and Kanemura Y.

359 2009. ABCB1 is predominantly expressed in human fetal neural stem/progenitor cells at an early

360 development stage. Journal of Neuroscience Research 87:2615-2623. 10.1002/jnr.22094

361 Yu J, Vodyanik MA, Smuga-Otto K, Antosiewicz-Bourget J, Frane JL, Tian S, Nie J, Jonsdottir

362 GA, Ruotti V, Stewart R, Slukvin, II, and Thomson JA. 2007. Induced pluripotent stem cell lines

363 derived from human somatic cells. Science 318:1917-1920. 10.1126/science.1151526 


\section{Figure Legends}

366 Fig. 1. Drug-screening strategies.

367 (a) Schematic of endpoint assay (ATP assay) and time-course cytotoxicity test. Cells were

368 cultured in a standard 96-well plate for the endpoint assay and in a microsphere array (MSA) for

369 the time-course cytotoxicity test. Same-colored circles indicate the same microwells in the panels

370 for days $0,1,2,3$, and 7. (b) Representative phase-contrast images taken on days $0,1,2,3$, and 7.

371 One field contains approximately 31 microwells. Same-colored circles indicate the same

372 microwells during the test. Black areas are the projected areas of neurospheres, which consist of

373 viable cells. The * on the day-7 panel shows the locations of dead cells around the neurosphere.

374 Scale bar, $500 \mu \mathrm{m}$.

375

376

Fig. 2. Dose-response curves and $\mathrm{IC}_{50}$ values obtained from a conventional ATP assay on

377 day 2.

378 The $\mathrm{x}$-axis indicates the drug concentration $(\mu \mathrm{M})$ in log scale, and the $\mathrm{y}$-axis indicates ATP

379 levels in the treated cells relative to the DMSO control (\%). Colors indicate cell type. The log-

380 logistic model (LL2.4) was used. Error bars represent the 95\% CI.

382 Fig. 3. Results of time-course cytotoxicity test. 
383 The $\mathrm{x}$-axis indicates days after treatment, and the y-axis indicates neurosphere size relative to the

384 DMSO control (\%). Colors indicate drug concentrations. The logistic model (L.4) was used.

385 Error bars represent the $95 \% \mathrm{CI}$.

386

387 Table $1 . \mathrm{IC}_{50}$ values $(\boldsymbol{\mu M})$ of drugs against hiPSC-NSPCs and $\mathrm{hN}-\mathrm{NSPCs}$

388

389 Fig. S1 (related to Fig. 3).

390 Time-course of changes in neurosphere size relative to day 0 neurospheres $(\%)$. Colors indicate

391 drug concentrations. The Brain-Cousens five-parameter model (BC.5) was used. Error bars

392 represent the $95 \% \mathrm{CI}$.

393

394 Table S1 (related to Fig. 3 and Fig. S1).

395 The number of neurospheres used in the time-course cytotoxicity test

396

397 Table S2 (related to Fig. 2).

398 Raw data on ATP content

399 Table S3 (related to Fig. 3 and Fig. S1).

400 Raw data on projected areas of neurosphere size obtained by time-course cytotoxicity test 


\section{Figure 1 (on next page)}

Drug-screening strategies.

(a) Schematic of endpoint assay (ATP assay) and time-course cytotoxicity test. Cells were cultured in a standard 96-well plate for the endpoint assay and in a microsphere array (MSA) for the time-course cytotoxicity test. Same-colored circles indicate the same microwells in the panels for days $0,1,2,3$, and 7. (b) Representative phase-contrast images taken on days $0,1,2,3$, and 7 . One field contains approximately 31 microwells. Same-colored circles indicate the same microwells during the test. Black areas are the estimated areas of neurospheres, which consist of viable cells. The * on the day-7 panel shows the locations of dead cells around the neurosphere. Scale bar, $500 \mu \mathrm{m}$. 


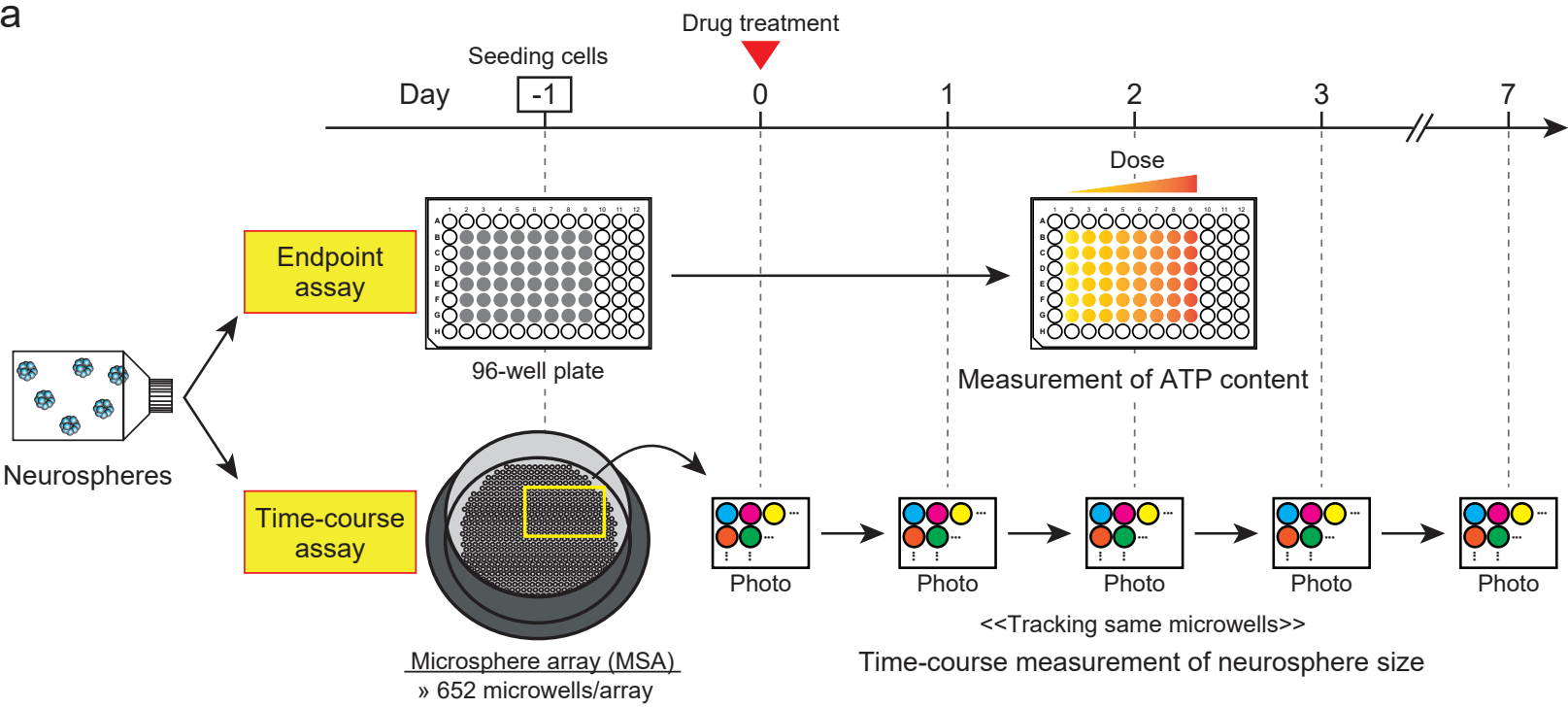

b

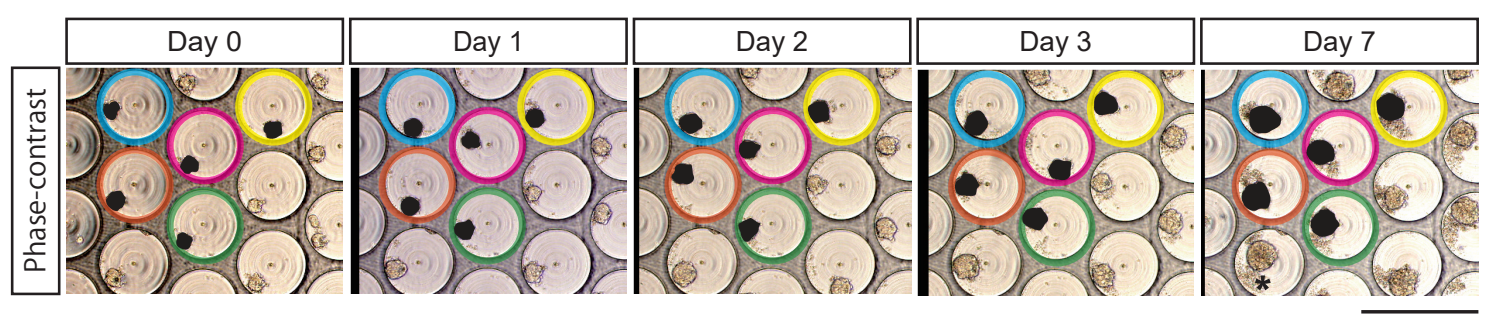




\section{Figure 2 (on next page)}

Dose-response curves and $\mathrm{IC}_{50}$ values obtained from a conventional ATP assay on day 2. control (\%).

The $x$-axis indicates the drug concentration $(\mu \mathrm{M})$ in log scale, and the $y$-axis indicates ATP levels in the treated cells relative to the DMSO control (\%). Colors indicate cell type. The loglogistic model (LL2.4) was used. Error bars represent the $95 \% \mathrm{Cl}$. 

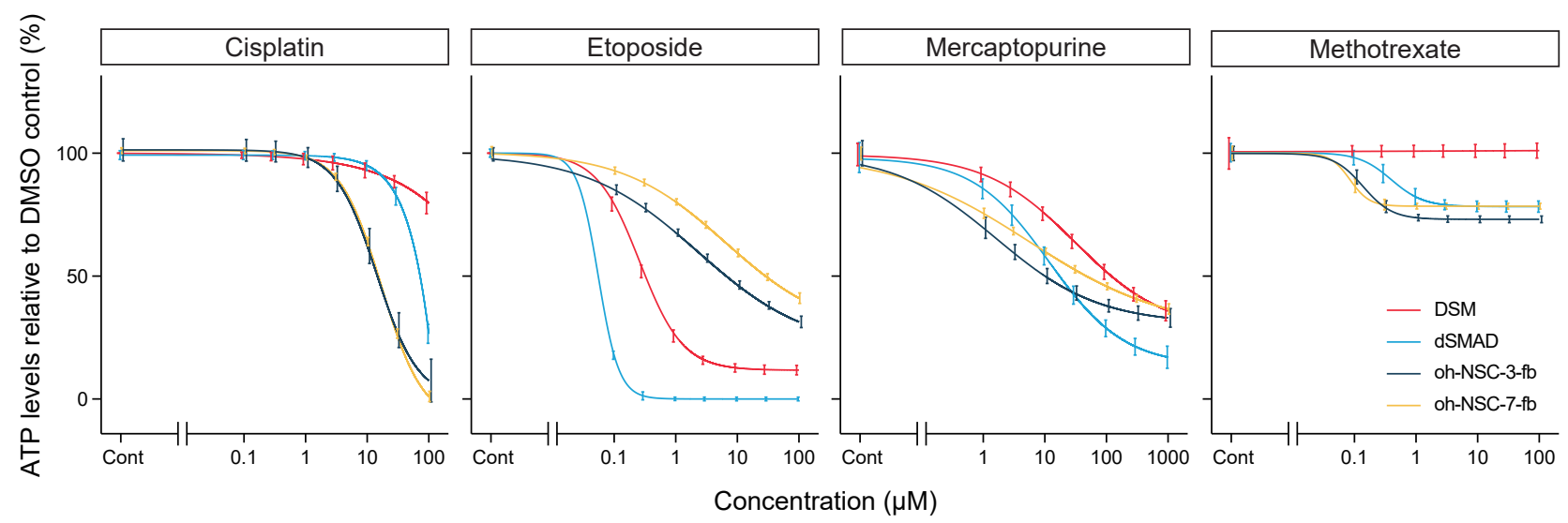


\section{Figure 3 (on next page)}

Results of time-course cytotoxicity test.

The $\mathrm{x}$-axis indicates days after treatment, and the $\mathrm{y}$-axis indicates neurosphere size relative to the DMSO control (\%). Colors indicate drug concentrations. The logistic model (L.4) was used. Error bars represent the $95 \% \mathrm{Cl}$. 


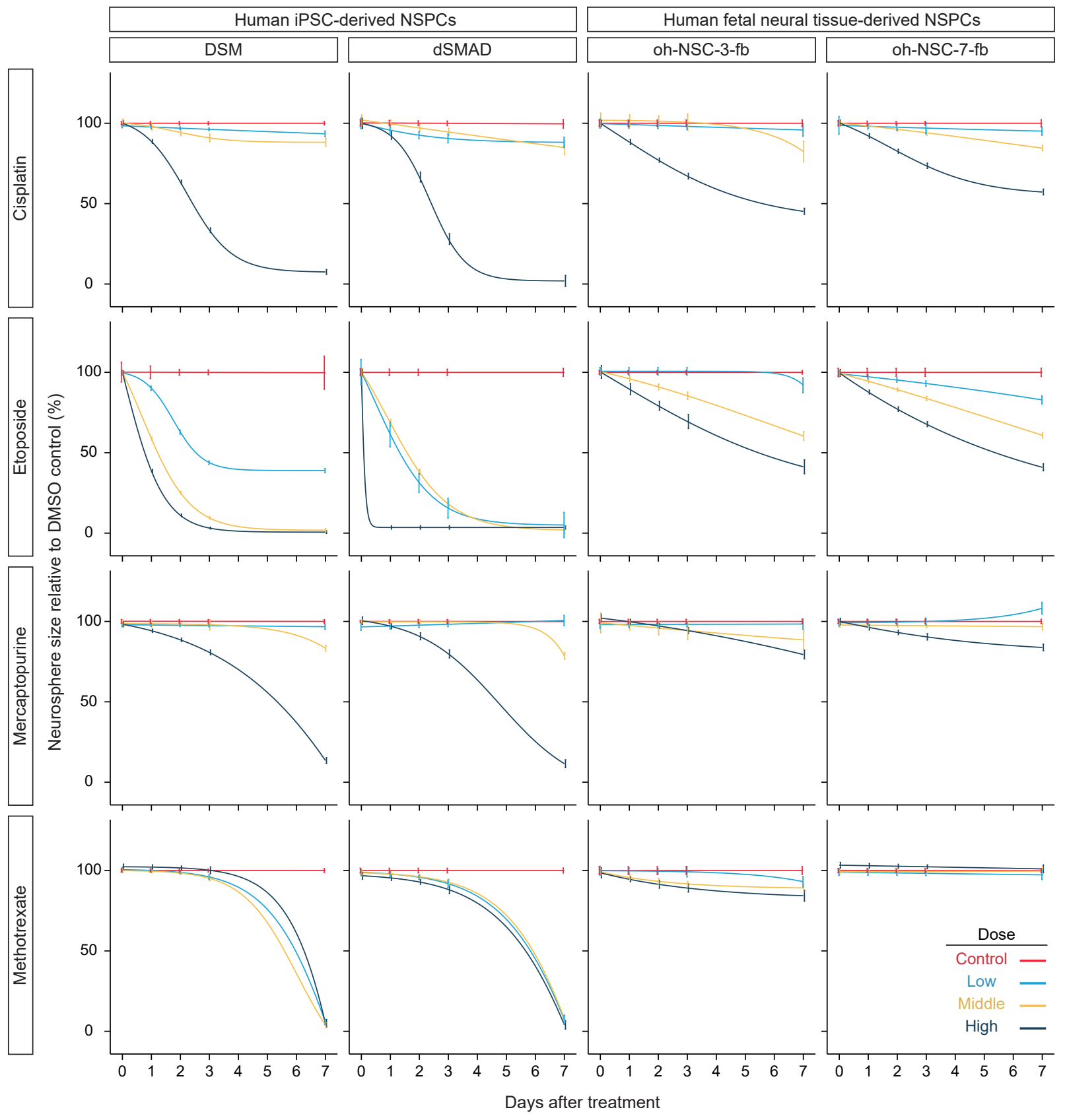




\section{Table $\mathbf{1}$ (on next page)}

$\mathrm{IC}_{50}$ values $(\mu \mathrm{M})$ of drugs against hiPSC-NSPCs and hN-NSPCs 
1 Table 1. $\mathrm{IC}_{50}$ values $(\mu \mathrm{M})$ of drugs against hiPSC-NSPCs and hN-NSPCs

\begin{tabular}{|c|c|c|c|c|}
\hline \multirow{2}{*}{ Drug } & \multicolumn{2}{|c|}{ hiPSC-NSPCs } & \multicolumn{2}{|c|}{ hN-NSPCs } \\
\hline & DSM & dSMAD & oh-NSC-3-fb & oh-NSC-7-fb \\
\hline Cisplatin & $100<$ & 72.3 & 14.6 & 15.2 \\
\hline Etoposide & 0.32 & 0.04 & 6.59 & 28.5 \\
\hline Mercaptopurine & 120 & 17.3 & 17.3 & 47.0 \\
\hline Methotrexate & $100<$ & $100<$ & $100<$ & $100<$ \\
\hline
\end{tabular}

2 